\title{
A comparison of cognitive function, sleep and activity levels in disease-free breast cancer patients with or without cancer-related fatigue syndrome
}

\author{
Ollie Minton, Patrick C Stone
}

Division of Population Health Sciences and Education, St George's, University of London, London, UK

\section{Correspondence to}

Dr Ollie Minton, Division of Population Health Sciences and Education, 6th floor Hunter Wing, St George's, University of London, Cranmer Terrace, London SW17 ORE, UK; ominton@sgul.ac.uk

Received 23 November 2011 Accepted 20 April 2012 Published Online First 31 May 2012

\section{ABSTRACT}

Background Chronic fatigue is a feature in a subset of women successfully treated for breast cancer but is not well characterised. This study examines differences in objective cognitive function, activity levels and sleep in disease-free women who do and do not meet criteria for cancer-related fatigue syndrome (CRFS).

Methods Women between 3 months and 2 years after completion of any primary therapy were recruited from a cancer centre follow-up clinic. On the basis of a diagnostic semi-structured interview they were classified as being CRFS cases or non-fatigued controls. Participants underwent objective cognitive testing using a computerised battery, wore an activity monitor for 1 week and completed quality of life and fatigue questionnaires.

Results 114 women were recruited (69 controls and 45 CRFS cases). There were significant differences between groups on fatigue, mood, sleep and quality of life scores, and in objective cognitive testing (tests of sustained attention, reaction time and verbal memory all $p<0.03)$. There was an overall difference in daytime activity $(p=0.03)$ from actigraphy recordings. There were no differences on objective measures of sleep or in routine laboratory measures.

Conclusions Our preliminary results suggest that disease-free women with CRFS after successful breast cancer treatment have significantly lower subjective quality of life and mood. Additionally, objective cognitive impairment in certain domains may play an important role in the subjective manifestation of these symptoms. There is also objective evidence on actigraphy of differing levels of activity. The subjective sleep disturbance and higher prevalence of insomnia do not correlate with objective measures.

\section{INTRODUCTION}

Two thirds of women diagnosed with breast cancer today are likely to survive for at least 20 years. ${ }^{1}$ As a result more women are living with the longerterm side-effects of treatment. These can include neurocognitive changes, ${ }^{2}{ }^{3}$ sleep disturbance, ${ }^{4}$ quality of life impairment and persistent fatigue. ${ }^{5}$ Of the many symptoms associated with cancer and its treatment, fatigue is probably the most under-recognised and poorly managed. ${ }^{6}$

We have previously undertaken a systematic review of the literature regarding the prevalence of fatigue in breast cancer survivors (BCS). ${ }^{7}$ Many of the early studies of fatigue in cancer populations were limited by the use of poorly validated assessment instruments and the lack of a coherent definition of cancer-related fatigue syndrome (CRFS). ${ }^{8}$ However, in 1998 Cella and colleagues ${ }^{9}$ proposed the introduction of diagnostic criteria for CRFS, which have now been successfully applied in a number of clinical studies. ${ }^{10-14}$ These criteria are based on similar principles to those used to diagnose patients with chronic fatigue syndrome (CFS). ${ }^{15}$ Most recently, our own group rigorously applied the diagnostic criteria to BCS between 3 months and 2 years after completion of treatment. We found the prevalence of CRFS to be $30 \%{ }^{16}$

CRFS is a clinical diagnosis and as such relies on the subjective reports of patients. Broadly speaking, patients' complaints of fatigue relate to either decrements in motor performance (physical fatigue) or impaired cognitive abilities (mental fatigue). Unfortunately, the same term can be used to describe both an objective physical or mental decrement in performance and a subjective mental state. Both types of fatigue are usually found to a greater or lesser extent in the same individual. However, there is no direct correspondence between the two phenomena. It is quite possible to feel extremely subjectively fatigued but to perform relatively normally on objective tests of physical or mental functioning.

However, patients' subjective complaints of physical fatigue relate to changes in the activities of daily living, mobility and the need for daytime naps. Unobtrusive objective monitoring is required to investigate these phenomena and studies have been performed using actigraphy (real-time activity monitoring). Overall, the data from several studies ${ }^{17} 18$ suggest that there is a strong correlation between changes in subjective fatigue and actigraphy data measures. The most consistent correlations occur between fatigue measures and disruption of sleep quality. However, the limitations of and variations in data collection mean these findings need further confirmation. In particular, these data have been recorded in patients on treatment and linked to sleep disturbances and have not adequately assessed the level of daytime fatigue. The exception to this was a study by our group $^{16}$ in which, contrary to expectation, we found no significant differences in activity levels in a population of fatigued BCS compared to nonfatigued controls.

Several researchers have investigated cognitive changes in cancer survivors. Recent systematic reviews ${ }^{19}$ and a meta-analysis ${ }^{3}$ concluded that there is some evidence of objective declines in 
cognitive function (particularly verbal memory and executive function) following chemotherapy, but only one previous study has attempted to relate these objective findings to subjective complaints of fatigue. ${ }^{20}$ However, the authors did not use the diagnostic criteria ${ }^{9}$ and only employed a limited range of cognitive testing.

The primary aim of this study was to examine differences in objective measures of cognition (specifically focusing on previously identified cognitive domains that were affected) and in physical activity in women who did or did not meet the criteria for CRFS.

\section{MATERIALS AND METHODS}

BCS were recruited from January 2009 to May 2011 from a single centre nurse-led follow-up clinic at St George's Healthcare NHS Trust. Approval was obtained from Wandsworth ethics committee prior to data collection (ref 08/H0803/182).

All patients who were clinically and radiologically diseasefree between 3 months and 2 years after the end of their primary treatment (of any modality) were invited to participate. Concurrent hormone use was allowed.

Those patients with significant cognitive impairment, a psychiatric history or medical co-morbidities on notes review or initial discussion with the researcher $(O M)$ were excluded from entering the study ( 12 women in total). This was a pragmatic decision as these women were not eligible for the complete study.

Eligible women were identified from the clinic list 14 days before their appointment and received an introductory letter and an information sheet by post. Women who declined to participate in the study were asked if they would be willing to complete a short fatigue questionnaire.

\section{Assessment 1}

Women who consented to participate were invited to an initial consultation in a designated research facility and completed the following interviews and actigraphy.

\section{Diagnostic Interview for Cancer-Related Fatigue}

This interview determines whether the participant meets the four criteria for a diagnosis of $\mathrm{CRFS}^{9}$ : criterion A-the presence of 2 weeks of significant fatigue in the preceding month and the presence of at least five out of nine other fatiguerelated symptoms; criterion $\mathrm{B}$ - the fatigue has a significant effect on work or self-care; criterion C-the fatigue symptoms are a consequence of cancer or cancer therapy; and criterion $\mathrm{D}$ - the symptoms are not primarily a consequence of a comorbid psychiatric disorder. The final criterion can be assessed clinically, but the most robust method is to use a contemporaneous psychiatric interview. Participants with a significant psychiatric history which was felt to be contributing to fatigue were excluded.

\section{Structured Clinical Interview for the DSM-IV (SCID)}

The Structured Clinical Interview for the DSM-IV (SCID) provides a method for obtaining Diagnostic and Statistical Manual (DSM)-IV diagnoses. The procedure has been successfully used in previous studies examining CRFS ${ }^{11} 12$ and by our group. ${ }^{16}$ All interviews were conducted by the same person (OM). The SCID can be administered by a non-psychiatrist. OM was trained in administration and supervised by a consultant liaison psychiatrist. The average administration time was $30 \mathrm{~min}$.

\section{Other questionnaires}

All women were also asked to complete the following questionnaires:

(1) Functional Assessment of Cancer Therapy (FACT-F). This is a 13 -item questionnaire to measure fatigue ${ }^{21}$ in cancer and has been widely used in cancer treatment trials. ${ }^{22}$

(2) The Chalder Fatigue Scale (FS). ${ }^{23}$ This is an 11-item questionnaire to measure fatigue. It was originally developed in non-cancer patients but has been extensively used in a number of studies in cancer. ${ }^{24}$

(3) All participants were asked to give a score of average fatigue over the last week on a $0-10$ visual analogue scale (VAS-F), where 0 is no fatigue and 10 is fatigue as bad as you can imagine (after National Comprehensive Cancer Network (NCCN) guidance). ${ }^{25}$

(4) Hospital Anxiety and Depression Scale (HADS). This is a 14-item scale with seven items on anxiety and seven items on depression. ${ }^{26}$ It is used extensively in clinical trials and has been employed by our group previously in fatigue assessment studies. ${ }^{27}$

(5) European Organisation of Research and Treatment of Cancer Quality of Life Questionnaire (EORTC QLQ-30). This is a 30 -item instrument with five functional scales, three symptom scales and a global quality of life score. ${ }^{28}$

(6) EORTC Breast Module (BR23). This is a 23-item scale which is validated for use in breast cancer patients. ${ }^{29}$ It includes items on breast and arm symptoms and systemic treatment effects.

(7) The 7-item Insomnia Severity Index (ISI). ${ }^{30}$ This scale has previously been validated in a breast cancer population. ${ }^{31}$ The seven items in this scale were combined with two additional sleep related questions to determine if insomnia diagnostic criteria were met:

- During the past month how many nights per week have you taken more than 30 min to fall asleep?

- During the past month how many nights per week were you awake for more than $30 \mathrm{~min}$ ?

A diagnosis of insomnia syndrome was made if one of the above two features was present on more than three nights per week on average, there was significant distress as scored on the ISI and/or fatigue or daytime somnolence was present. It took $20 \mathrm{~min}$ on average to administer these questionnaires and conduct the CRFS interview. An initial appointment time of an hour was made and potential participants were made aware of this time requirement.

\section{Actigraphy}

After completion of the questionnaires and interview, participants were asked to wear an Actiwatch AW4 (CamNtech, Cambridge, UK) for a period of 7 days and nights. The associated software and sleep algorithm ${ }^{32}$ allows for the determination of selected measures of activity, sleep and circadian rhythm (over a 7-day average). A predetermined $12 \mathrm{~h}$ timeframe is used to define daytime (light) activity: 06:00 to 18:00 h. The night-time activity period (dark) is defined as 18:00 to 06:00 h. The participants used an event marker on the watch to record their own individualised sleep patterns. This was recorded as the time entering and exiting bed overnight and did not include naps. 


\section{Assessment 2}

Participants underwent objective cognitive testing using a predetermined computerised battery approximately 1 week later (CANTABeclipse, Cambridge Cognition, Cambridge, UK). The procedure uses a touch screen computer (Slimbook P210; PaceBlade, Amersfoort, The Netherlands) and press pad. It consists of seven different tests designed to assess the cognitive domains thought to be affected by previous breast cancer treatment. ${ }^{19}$ This computerised battery has been previously used in a longitudinal study of cognitive changes after breast cancer treatment. ${ }^{33}$ The procedure takes 45 min to complete and was administered by one of the authors (OM).

The tests and cognitive domains studied are listed below:

- Paired Associates Learning (PAL; visuospatial ability and visual memory)

- Rapid Visual Information Processing (RVP; sustained attention and reaction time - motor skills)

- Match to Sample Visual Search (MTS; visuospatial ability and motor skills)

- Verbal Recognition Memory (VRM; verbal memory)

- Delayed Matching to Sample (DMS; attention)

- Affective Go No-go (AGN; decision and response and information processing)

- Intra-Extra Dimensional Set Shift (IED, executive function/information processing).

\section{Statistical considerations}

The power calculation was based on an estimated sample size of 96 women. With 48 cases and 48 controls, the study would have had $80 \%$ power to detect a 0.57 (medium) betweengroup effect size on questionnaire and objective monitoring data at a $5 \%$ significance level. Since this was an exploratory and hypothesis generating study, no correction was made for multiple analyses. The between-group analysis was conducted using t tests with comparisons between mean scores.

\section{RESULTS}

Due to time and resource constraints, recruitment of fatigued patients terminated slightly early with 45 of an expected 48 patients recruited. However, the number of control subjects was larger than required. A total of 114 women completed the study (69 controls and 45 cases of CRFS). Non-participants $(\mathrm{NP}=102)$ were asked to complete the Chalder fatigue questionnaire and ethics approval was obtained to collect anonymised demographic and treatment data from these women. The main reasons for non-participation were cited as work (28\%) or the need for extra visits (32\%), while $12 \%$ mentioned other medical problems and the remainder either had 'had enough' or did not want to think about their cancer (28\%). The NP had a significantly lower fatigue score (10.7 vs $13.1 ; \mathrm{p}=0.04)$ and were older (62 years vs 57 years; $p=0.005$ ) than the participants. There were no differences on treatment variables, stage at diagnosis or time since diagnosis.

\section{Cases versus controls}

The overall prevalence of CRFS was 39\% (45/114). A between-group comparison for all data is shown in tables 1-5 (table 1: treatment variables; table 2: questionnaire data; table 3: routine laboratory parameters; table 4: actigraphy data; and table 5: cognitive testing data).

There were no significant differences between groups in mean age (CRFS 56.0 years vs controls 58.6 years; $p=0.17$ ), time since treatment (CRFS 12.4 months vs controls 13.0 months; $\mathrm{p}=0.67$ ) or body mass index (CRFS $27.1 \mathrm{~kg} / \mathrm{m}^{2}$ vs controls 26.0 $\mathrm{kg} / \mathrm{m}^{2} ; \mathrm{p}=0.29$ ).

There were no significant differences in treatment variables between groups (table 1).

There was a significantly higher prevalence of oestrogen receptor negative tumours in the control group. There was no difference in the percentage of non-white participants between groups.

There were significant differences in the levels of fatigue, mood disturbance and quality of life. There were also a number of differences in symptom subscales on both the EORTC QLQ-30 and BR23 (table 2).

The prevalence of insomnia syndrome was significantly higher in the CRFS group (44\% vs $16 \%$; $=0.001$ ).

The routine laboratory variables (table 3 ), measured to exclude any gross metabolic disturbance, did not show any significant differences between groups.

The actigraphy data (table 4) which provided a measure of sleep, activity and circadian rhythm, demonstrated some differences. There was a minor difference in sleep with the control group actually having significantly shorter sleep bouts. However, there were no differences in the major sleep parameters (overall time or quality). There were significant differences in average daytime activity and duration of daytime (light) activity.

The cognitive data (table 5) demonstrated subtle but important differences between the groups. Significant differences

Table 1 Treatment and pathological variables

\begin{tabular}{lllllll}
\hline & \multicolumn{2}{l}{ CRFS cases } & & & Controls & \\
Treatment variable & Frequency & Percentage & & Frequency & Percentage & p Value \\
\hline Conserving surgery & 31 & 69 & 40 & 58 & 0.24 \\
Non-conserving surgery & 14 & 31 & 29 & 42 & \\
Chemotherapy & 19 & 42 & 35 & 51 & 0.37 \\
No chemotherapy & 26 & 58 & 34 & 49 & \\
Radiotherapy & 39 & 87 & 51 & 74 & 0.10 \\
No radiotherapy & 6 & 13 & 18 & 26 & \\
Hormone therapy & 6 & 13 & 11 & 16 & 0.79 \\
No hormone therapy & 39 & 87 & 58 & 84 & \\
Lymph node positive & 17 & 38 & 17 & 25 & 0.13 \\
Lymph node negative & 28 & 62 & 52 & 75 & \\
Oestrogen receptor positive & 42 & 93 & 54 & 78 & 0.03 \\
Oestrogen receptor negative & 3 & 7 & 15 & 22 & \\
HER2 receptor positive & 6 & 13 & 8 & 12 & 0.78 \\
HER2 Receptor negative & 39 & 87 & 61 & 88 & \\
\hline
\end{tabular}

CRFS, cancer-related fatigue syndrome. 
Table 2 Questionnaire data

\begin{tabular}{|c|c|c|c|c|c|}
\hline \multirow[b]{2}{*}{ Variable } & \multicolumn{2}{|c|}{ CRFS cases } & \multicolumn{2}{|c|}{ Controls } & \multirow[b]{2}{*}{ p Value } \\
\hline & Mean & SD & Mean & SD & \\
\hline FS total & 18.87 & 5.35 & 9.22 & 6.35 & $<0.001$ \\
\hline FACT-F total & 26.84 & 8.07 & 14.54 & 6.83 & $<0.001$ \\
\hline ISI total & 13.96 & 5.99 & 9.01 & 5.98 & $<0.001$ \\
\hline HADS total & 14.82 & 6.47 & 7.26 & 5.03 & $<0.001$ \\
\hline VAS-F scale & 6.44 & 1.53 & 4.73 & 2.29 & $<0.001$ \\
\hline \multicolumn{6}{|l|}{ EORTC OLQ-30 subscales } \\
\hline Physical functioning & 69.19 & 19.45 & 85.80 & 14.00 & $<0.001$ \\
\hline Global health status & 81.09 & 16.80 & 56.07 & 22.79 & $<0.001$ \\
\hline Emotional functioning & 63.70 & 20.73 & 80.80 & 17.98 & $<0.001$ \\
\hline Cognitive functioning & 56.67 & 23.41 & 79.47 & 19.63 & $<0.001$ \\
\hline Social functioning & 59.26 & 27.65 & 84.81 & 19.69 & $<0.001$ \\
\hline Fatigue & 55.80 & 21.52 & 25.44 & 17.68 & $<0.001$ \\
\hline Nausea/vomiting & 7.04 & 13.98 & 2.42 & 6.57 & 0.04 \\
\hline Pain & 37.04 & 33.88 & 16.91 & 21.86 & 0.01 \\
\hline Dyspnoea & 22.96 & 27.36 & 11.59 & 19.66 & 0.02 \\
\hline Insomnia & 48.15 & 32.22 & 37.68 & 32.29 & 0.09 \\
\hline Appetite loss & 11.85 & 23.74 & 7.25 & 14.98 & 0.25 \\
\hline Constipation & 12.59 & 23.88 & 6.28 & 14.32 & 0.15 \\
\hline Diarrhoea & 2.96 & 9.59 & 3.86 & 13.45 & 0.68 \\
\hline Financial difficulties & 31.85 & 34.78 & 9.18 & 20.52 & $<0.001$ \\
\hline \multicolumn{6}{|l|}{ EORTC BR23 subscales } \\
\hline $\begin{array}{l}\text { Breast systemic therapy side } \\
\text { effects }\end{array}$ & 30.37 & 16.22 & 16.25 & 11.53 & $<0.001$ \\
\hline Breast symptoms & 38.89 & 18.63 & 20.05 & 16.69 & $<0.001$ \\
\hline Arm symptoms & 32.59 & 29.24 & 12.91 & 16.25 & $<0.001$ \\
\hline
\end{tabular}

The VAS-F is a visual analogue fatigue scale from 0 to 10 . Values indicate average fatigue over the last 7 days. The EORTC QLQ-30 (European Organisation for Research and Treatment of Cancer 30-item Quality of Life Questionnaire) has defined subscales of functioning and symptom scores. Higher scores on symptom subscales indicate greater symptom severity, and higher scores on functional subscales indicate better functioning. The EORTC BR23 is a breast specific module with functioning and symptom scales.

CRFS, cancer-related fatigue syndrome; FACT-F, Functional Assessment of Cancer Therapy - fatigue subscale; FS, Chalder Fatigue Scale; HADS, Hospital Anxiety and Depression Scale; ISI, Insomnia Severity Index.

Table 3 Routine laboratory data

\begin{tabular}{|c|c|c|c|c|c|c|}
\hline \multirow[b]{2}{*}{ Variable } & \multirow{2}{*}{$\begin{array}{l}\text { Laboratory } \\
\text { reference range }\end{array}$} & \multicolumn{2}{|c|}{ CRFS cases } & \multicolumn{2}{|c|}{ Controls } & \multirow[b]{2}{*}{ p Value } \\
\hline & & Mean & SD & Mean & SD & \\
\hline Haemoglobin (g/dl) & $12-16$ & 12.9 & 0.78 & 12.9 & 0.91 & 0.94 \\
\hline White cell count $(\times 109 / I)$ & $4-11$ & 5.89 & 1.51 & 6.12 & 2.1 & 0.26 \\
\hline Neutrophil $(\times 109 / I)$ & $1.7-8.0$ & 3.54 & 1.08 & 4.41 & 4.7 & 0.17 \\
\hline Lymphocyte (×109/I) & $1.0-4.0$ & 1.84 & 0.71 & 1.80 & 0.68 & 0.79 \\
\hline Monocyte $(\times 109 / I)$ & $0.24-1.1$ & 0.36 & 0.16 & 0.37 & 0.12 & 0.60 \\
\hline Platelet $(\times 109 / I)$ & $150-450$ & 274 & 55.5 & 269 & 53.7 & 0.63 \\
\hline Sodium (mmol/l) & $135-145$ & 139.27 & 2.07 & 139.52 & 2.22 & 0.55 \\
\hline Potassium (mmol/l) & $3.5-4.7$ & 4.18 & 0.34 & 4.20 & 0.23 & 0.744 \\
\hline Urea (mmol/l) & $2.5-8.0$ & 5.48 & 1.19 & 5.74 & 1.53 & 0.32 \\
\hline Creatinine $(\mu \mathrm{m} / \mathrm{l})$ & $60-110$ & 64.10 & 8.29 & 66.59 & 8.20 & 0.13 \\
\hline Uncorrected calcium (mmol/l) & $2.18-2.47$ & 2.36 & 0.099 & 2.37 & 0.099 & 0.56 \\
\hline Adjusted calcium (mmol/l) & NA & 2.38 & 0.085 & 2.40 & 0.085 & 0.31 \\
\hline Phosphate (mmol/l) & $0.75-1.50$ & 1.12 & 0.15 & 1.08 & 0.20 & 0.24 \\
\hline Alkaline phosphatase (IU/I) & $30-100$ & 74.07 & 21.86 & 67.58 & 19.56 & 0.12 \\
\hline Alanine transaminase (IU/I) & $0-40$ & 25.16 & 11.16 & 27.72 & 14.17 & 0.32 \\
\hline Bilirubin $(\mu \mathrm{mol} / \mathrm{l})$ & $0-17$ & 6.81 & 3.15 & 7.35 & 2.98 & 0.37 \\
\hline Albumin (g/l) & $35-48$ & 38.67 & 2.90 & 38.56 & 2.06 & 0.83 \\
\hline GGT (IU/I) & $0-30$ & 29.33 & 23.54 & 30.49 & 24.92 & 0.81 \\
\hline TSH (mU/l) & $0.4-4.0$ & 2.63 & 3.31 & 3.25 & 5.05 & 0.50 \\
\hline Free T4 (pmol/l) & $12-24$ & 13.36 & 4.17 & 13.04 & 3.14 & 0.67 \\
\hline Magnesium (mmol/l) & $0.74-1.03$ & 0.86 & 0.68 & 0.86 & 0.08 & 0.74 \\
\hline
\end{tabular}

were found on the RVP and VRM tests. The AGN test, a sensitive measure of executive function, demonstrated a higher number of commissions (ie, incorrect responses) in the CRFS group. The CRFS group performed worse than the controls in all statistically significantly different tests.

\section{DISCUSSION}

This study is the first to objectively characterise cognitive differences between a rigorously defined group of cases of CRFS and a group of non-fatigued BCS. We found no differences in sleep actigraphy despite a much higher prevalence of insomnia 
Table 4 Actigraphy data

\begin{tabular}{|c|c|c|c|c|c|}
\hline \multirow[b]{2}{*}{ Variable } & \multicolumn{2}{|c|}{ CRFS cases } & \multicolumn{2}{|l|}{ Controls } & \multirow[b]{2}{*}{ p Value } \\
\hline & Mean & SD & Mean & SD & \\
\hline \multicolumn{6}{|l|}{ Sleep } \\
\hline Actual sleep time (h) & 06:04 & $00: 44: 41$ & 06:07 & $00: 45: 56$ & 0.74 \\
\hline Actual sleep (\%) & 84.40 & 6.41 & 82.49 & 10.28 & 0.27 \\
\hline Actual wake time (h) & 01:07 & $00: 28: 29$ & 01:11 & 00:30:31 & 0.48 \\
\hline Actual wake (\%) & 17.51 & 10.27 & 15.60 & 6.41 & 0.28 \\
\hline Sleep efficiency (\%) & 80.22 & 7.73 & 78.44 & 13.28 & 0.43 \\
\hline Sleep latency (min) & $00: 17: 32$ & $00: 20: 27$ & 00:17:19 & $00: 27: 36$ & 0.97 \\
\hline $\begin{array}{l}\text { Average number of calculated sleep } \\
\text { bouts }\end{array}$ & 23.73 & 6.60 & 24.04 & 7.02 & 0.82 \\
\hline $\begin{array}{l}\text { Average number of calculated wake } \\
\text { bouts }\end{array}$ & 23.77 & 6.68 & 24.10 & 7.02 & 0.81 \\
\hline Mean sleep bout time (min) & 00:33:03 & $00: 44: 41$ & $00: 19: 20$ & 00:13:41 & 0.02 \\
\hline \multicolumn{6}{|l|}{ Activity } \\
\hline Mean wake bout time (min) & $00: 02: 43$ & 00:00:42 & 00:03:25 & $00: 00: 28$ & 0.19 \\
\hline $\begin{array}{l}\text { Average value of epoch intensity } \\
(24 \mathrm{~h} \text { period })\end{array}$ & 208.48 & 87.22 & 245.82 & 96.32 & 0.04 \\
\hline $\begin{array}{l}\text { Difference in maximum and } \\
\text { minimum activity }\end{array}$ & 198.85 & 69.70 & 218.55 & 88.97 & 0.45 \\
\hline Peak intensity of epoch & 11.91 & 5.02 & 11.99 & 4.68 & 0.22 \\
\hline Average of daytime (light) activity & 299.25 & 131.18 & 342.92 & 119.92 & 0.03 \\
\hline Time of night time (dark) activity & 117.70 & 55.24 & 148.72 & 90.60 & 0.14 \\
\hline Ratio of light to dark activity & 2.99 & 1.54 & 2.99 & 1.54 & 0.82 \\
\hline \multicolumn{6}{|l|}{ Circadian rhythm } \\
\hline Interdaily stability & 0.53 & 0.12 & 0.57 & 0.15 & 0.16 \\
\hline Interdaily variability & 0.86 & 0.21 & 0.83 & 0.21 & 0.14 \\
\hline Least 5 (L5) active hours & 1475 & 1010 & 2348 & 1634 & 0.50 \\
\hline Most 10 (M10) active hours & 22481 & 7774 & 24865 & 8722 & 0.50 \\
\hline Relative amplitude & 0.88 & 0.07 & 0.86 & 0.14 & 0.22 \\
\hline
\end{tabular}

Sleep efficiency (\%) is time asleep (as per algorithm) divided by total time in bed.

Sleep latency is time in bed before algorithm records the subject being asleep.

Average value of epoch intensity ( $24 \mathrm{~h}$ period): the recording is converted to and analysed as a cosine wave around a zero point.

Difference in maximum and minimum in activity corresponds to the difference between the peak and trough of the cosine wave.

Interdaily stability is a measure of consistency in daily activity over 7 days.

Interdaily variability is a measure of fragmentation of periods of rest and activity; normal value are $<1$ and calculated over 7 days.

$\mathrm{L} 5$ indicates the $5 \mathrm{~h}$ of calculated lowest activity over the 7-day average.

M10 indicates the $10 \mathrm{~h}$ of calculated greatest activity over the 7-day average.

Relative amplitude is a measure of the difference between $\mathrm{L} 5$ and $\mathrm{M} 10$ and thus an indication of overall level of activity over the 7-day period.

CRFS, cancer-related fatigue syndrome.

syndrome in the CRFS group. However, mean daytime activity was lower in the CRFS group. These findings raise important questions about the nature of CRFS and its relationship to objective deficits in activity and cognition.

The strengths of this study are the tight categorisation of post-treatment fatigue in BCS and the extensive cognitive and activity testing. However, the sample size is small and it is only a single centre study. The multiple analyses may have led to type I errors. This was a hypothesis generating study and was underpowered to detect all the identified differences between the groups, so the discussion and conclusions should be interpreted in that context. However, we feel these are interesting data and are further validated when placed in the context of previous findings.

The domains in which cognitive dysfunction occurs are the same as identified in previous reviews of breast cancer patients. ${ }^{19}$ The areas of executive function, processing speed and verbal memory are important for day-to-day tasks and may explain why these are associated with the subjective sensation of mental fatigue. Similar findings were found in a comparative study between subjects with CFS and cancer fatigue ${ }^{34}$ but not in a study comparing fatigued and non-fatigued
BCS. ${ }^{20}$ However, in the latter study it should be noted that the 'fatigued' BCS group were defined using a cut-off on a continuous fatigue scale (rather than using a case-based definition) and the cognitive testing only included measures of concentration and reaction time (rather than the more comprehensive battery of tests employed in the current study). The authors failed to find differences between the fatigued and the non-fatigued group on objective cognitive testing. The objective evidence of cognitive impairment that we identified in this study appears to be consistent with the questionnaire data. The Chalder Fatigue Scale and the QLQ-30 both assess perceived cognitive impairment, and the scores on both of these questionnaires were significantly worse in the CRFS group.

One limitation of the cognitive assessments undertaken in this study was that they were only recorded at the end of treatment and were not measured prospectively from baseline. However, we were not specifically examining cognitive change per se following treatment but comparing CRFS and matched controls. The purpose was to identify potential cognitive deficits in the CRFS group. The exclusion of psychiatric co-morbidities reinforces the hypothesis that CRFS is associated with a defined set of cognitive deficits that are unrelated 
Table 5 Cognitive testing data

\begin{tabular}{|c|c|c|c|c|c|}
\hline \multirow[b]{2}{*}{ Variable } & \multicolumn{2}{|c|}{ CRFS cases } & \multicolumn{2}{|l|}{ Controls } & \multirow[b]{2}{*}{ p Value } \\
\hline & Mean & SD & Mean & SD & \\
\hline DMS percentage correct (all delays) & 81.78 & 11.63 & 84.83 & 11.43 & 0.17 \\
\hline DMS mean correct latency (ms) & 3528 & 1151 & 3707 & 1416 & 0.48 \\
\hline IED stages completed & 7.40 & 2.40 & 7.33 & 2.40 & 0.89 \\
\hline IED total errors (adjusted) & 50.37 & 51.71 & 52.65 & 53.43 & 0.83 \\
\hline MTS mean correct reaction time & 3515 & 1143 & 3156 & 1137 & 0.10 \\
\hline MTS per cent correct & 94.32 & 6.10 & 93.38 & 5.78 & 0.41 \\
\hline PAL stages completed & 3.40 & 0.78 & 3.50 & 0.86 & 0.76 \\
\hline PAL total errors (adjusted) & 95.60 & 29.67 & 92.90 & 32.05 & 0.65 \\
\hline PAL total trials (adjusted) & 20.47 & 6.78 & 20.09 & 7.29 & 0.78 \\
\hline RVP A' (scores between 0 and 1 ) & 0.87 & 0.06 & 0.89 & 0.07 & 0.15 \\
\hline RVP total correct rejections & 236.69 & 25.83 & 245.53 & 14.95 & 0.02 \\
\hline RVP mean latency (ms) & 533 & 127 & 468 & 126 & 0.009 \\
\hline VRM free recall - total correct & 7.00 & 1.77 & 7.75 & 1.75 & 0.03 \\
\hline VRM recognition - total correct & 22.78 & 1.38 & 23.06 & 1.42 & 0.3 \\
\hline AGN mean correct latency (ms) & 532 & 71 & 517 & 61 & 0.24 \\
\hline AGN total omissions & 5.17 & 1.99 & 4.57 & 1.78 & 0.64 \\
\hline AGN total commissions & 7.76 & 1.75 & 6.57 & 1.72 & 0.03 \\
\hline
\end{tabular}

AGN: Affective Go No-go (information processing and reaction time). There are three outcomes: mean correct latency (time taken for a correct response), total omissions which are incorrect responses to a target word (ie, the subject not pressing the button when they should have) and total commissions which are the total number of incorrect responses to the distracter words (ie, pressing the button inappropriately).

DMS: Delayed Matching to Sample (memory and reaction time). There are two outcomes: overall correct responses (percentage) and latency of response (time taken to make correct response).

IED: Intra-Extra Dimensional Set Shift (rule acquisition and reversal). There are two outcomes: stages complete is the number successfully completed and total errors (adjusted for stages) is a measure of the efficiency of completing the test.

MTS: Match To Sample (matching test with speed/accuracy trade off). There are two outcomes: overall correct responses (percentage) and latency of response (time taken to make correct response).

PAL: Paired Associates Learning (visual memory and new learning). There are two outcomes: total errors (adjusted from stages completed) and total trials (number of attempts needed to complete task adjusted for early abortion). RVP: Rapid Visual Information Processing (sensitive measure of general performance). There are three outcomes: $A^{\prime}$ is a probability score of how good a participant is at detecting sequences, correct rejection is the number of times a false sequence is ignored between correct ones and the mean latency is the time of response between the end of a sequence and the participant pressing the button.

VRM: Verbal Recognition Memory (verbal information under free recall and forced choice recognitions). There are two outcomes: free recall number of correct words and a forced choice correct total (yes/no as to whether word was in the original list).

CRFS, cancer-related fatigue syndrome.

to mood disturbance. The underlying mechanism of cognitive dysfunction is unclear and may be related to an increase in circulating inflammatory cytokines ${ }^{35}$ but seems unlikely to be related to a gross hypothalamic pituitary axis disturbance. ${ }^{36}$ Functional imaging studies in this population would help to further elucidate the mechanism. The association between subjective fatigue and objective cognitive deficits is also interesting in relation to the finding that psychostimulants can reduce cancer fatigue during treatment. ${ }^{37}$ However, they are unlikely to be suitable for longer term use in $\mathrm{BCS}^{38}$ because of concerns related to adverse effects and the risk of addiction.

The higher prevalence of insomnia syndrome in the CRFS group was linked to higher scores on the ISI. However, this subjective perception of difficulty with sleeping did not correlate with objective actigraphy measures of sleep. While $44 \%$ of CRFS meet the criteria for insomnia syndrome, they are clearly two separate entities and CRFS cannot be explained by sleep disturbances alone. This finding is supported by data from an intervention trial for insomnia that improved sleep but not fatigue. ${ }^{39}$

We found significant differences between groups with respect to objective activity data. This is in keeping with a recent study ${ }^{40}$ in breast cancer but in conflict with our earlier findings. ${ }^{16}$ The reason for the disparity is unclear but may be due to the different actigraph used and associated analysis algorithm. The differences were seen in the average epoch score (a measure of movement intensity) and in the level of daytime activity. These data confirm that patients who fulfil the criteria for CRFS, as well as being subjectively more fatigued, also have evidence of decreased physical activity. The decreased activity may be a direct consequence of feeling subjectively fatigued, or the decreased activity may arise first (due to a variety of causes) and may be a causative factor in exacerbating or perpetuating the subjective fatigue that these patients experience. ${ }^{41}$ One way to disentangle these phenomena would be to undertake a longitudinal study to assess both subjective and objective manifestations of fatigue and to trace the temporal relationships between them. ${ }^{40}$ The authors of the study ${ }^{40}$ did not use diagnostic criteria to measure fatigue. The theory that, whatever the cause of the initial fatigue, it is perpetuated by physical deconditioning and decreased activity probably explains why exercise is reported to be an effective treatment for cancer related fatigue. ${ }^{42}$ It is likely, however, that the effects of exercise are relatively non-specific (exercise reduces fatigue even in healthy individuals) and this explains the relatively small effect size for exercise interventions in cancer populations.

In a previous study in BCS we found the prevalence of CRFS to be $30 \%{ }^{16}$ and this general figure was supported by the results of a systematic review. ${ }^{7}$ The prevalence of $39 \%$ for CRFS that we found in this study although slightly higher than previous 
estimates is broadly in keeping with these figures and may be explained by the finding that the non-participant group were significantly less fatigued ${ }^{16}$ than the study sample. A prevalence of $30-39 \%$ represents a sizeable percentage of women who have successfully completed treatment for breast cancer and potentially translates into a large absolute number of BCS. This emphasises that it is important to recognise and treat fatigue.

The widespread differences in questionnaire data and levels of other symptoms are in keeping with previous studies. ${ }^{12} \mathrm{We}$ found that the CRFS group had a higher level of mood disturbance despite the exclusion of patients with specific psychiatric diagnoses. It is possible that sub-threshold mood disturbance contributes to CRFS. However fatigue and depression should be regarded as separate entities. ${ }^{43}$

There are some important negative findings in this study. There were no significant differences in any of the routine laboratory tests. While fatigue may be linked to anaemia during chemotherapy, ${ }^{44}$ this does not appear to be the case in BCS. There is also no link to thyroid disturbances. We had postulated that serum magnesium levels may be contributory but this was not found to be the case.

There were also no differences in treatment or demographic variables between groups. This is consistent with a critical appraisal ${ }^{45}$ in which the authors found very limited associations between fatigue and these variables.

The clinical significance of these findings reinforces the importance of identifying women who have or are at risk of developing CRFS. This should be incorporated into routine practice and might include counselling on potential cognitive and sleep changes. These problems might be diminished with simple information giving at the start of treatment, ${ }^{46}$ which would in keeping with the Department of Health survivorship strategy. ${ }^{47}$ However, we are not able to recommend any particular treatment strategy at present in this group. ${ }^{7}$ In conclusion, we have found that a significant minority of women successfully treated for breast cancer meet the criteria for CRFS. This group is characterised by subjective poly-symptomology. There are important differences between the groups with objectively lower cognitive function and daytime activity in the CRFS group that need further confirmation with a larger sample size. Future work should examine this relationship longitudinally, possibly within a quality of life arm of a treatment trial. Correlation with levels of cytokines and/or functional imaging would be helpful to further characterise this group. The overall aim should be to design more targeted treatments to manage this problematic syndrome.

Contributors $\mathrm{OM}$ and PS both contributed equally to the manuscript. OM was responsible for data collection and analysis. OM and PS both obtained funding for the study.

Acknowledgements The authors would like to thank Sue Lowndes, Dr Cathy Coleman and Joe Diffley for their help in accessing the patients from the breast follow-up clinic they run. The authors would also like to thank Jo Passley and the other members of the St George's clinical research facility for their help in conducting the study. The authors also thank Dr Fiona Lofts, Mr Anup Sharma and Mr Dibeyshwar Banerjee for their help in setting up the study.

Funding This study was funded by Cancer Research UK Grant number C31193/ A10090.

Competing interests None.

Ethics approval Wandsworth research ethics committee approved this study (ref 08/H0803/182).

Provenance and peer review Not commissioned; externally peer reviewed.

\section{REFERENCES}

1. CRUK: Breast Cancer survival statistics http://info.cancerresearchuk.org/cancerstats/ types/breast/survival/ (ed), (accessed 28 February 2007).

2. Fan HG, Houédé-Tchen N, Yi OL, et al. Fatigue, menopausal symptoms, and cognitive function in women after adjuvant chemotherapy for breast cancer: 1- and 2-year follow-up of a prospective controlled study. J Clin Oncol 2005;23:8025-8032.

3. Taunk NK, Haffty BG, Chen $\mathrm{S}$, et al. Comparison of radiation-induced fatigue across 3 different radiotherapeutic methods for early stage breast cancer. Cancer 2011;117:4116-4124.

4. Fortner BV, Stepanski EJ, Wang SC, et al. Sleep and quality of life in breast cancer patients. J Pain Symptom Manage 2002;24:471-480.

5. Bower JE, Ganz PA, Desmond KA, et al. Fatigue in long-term breast carcinoma survivors: a longitudinal investigation. Cancer 2006;106:751-758.

6. Stone $\mathbf{P}$, Richardson A, Ream E, et al. Cancer-related fatigue: inevitable, unimportant and untreatable? Results of a multi-centre patient survey. Cancer Fatigue Forum. Ann Oncol 2000;11:971-975.

7. Minton 0, Stone P. How common is fatigue in disease-free breast cancer survivors? A systematic review of the literature. Breast Cancer Res Treat 2008;112:5-13.

8. Stone P. The measurement, causes and effective management of cancer-related fatigue. Int J Palliat Nurs 2002;8:120-128.

9. Cella D, Passik S, Jacobsen $P$, et al. Progress toward guidelines for management of fatigue. Oncology 1998;12:369-377.

10. Cella D, Davis K, Breitbart W, et al.; Fatigue Coalition. Cancer-related fatigue: prevalence of proposed diagnostic criteria in a United States sample of cancer survivors. J Clin Oncol 2001;19:3385-3391.

11. Sadler IJ, Jacobsen PB, Booth-Jones M, et al. Preliminary evaluation of a clinical syndrome approach to assessing cancer-related fatigue. J Pain Symptom Manage 2002;23:406-416.

12. Andrykowski MA, Schmidt JE, Salsman JM, et al. Use of a case definition approach to identify cancer-related fatigue in women undergoing adjuvant therapy for breast cancer. J Clin Oncol 2005;23:6613-6622.

13. Fernades R, Stone P, Andrews P, et al. Comparison between fatigue, sleep distrurbance, and circadian rhythm in cancer inpatients and healthy volunteers: evaluation of diagnostic criteria for cancer-related fatigue. J Pain and Symptom Manage 2006;32:245-254.

14. Murphy H, Alexander S, Stone P. Investigation of diagnostic criteria for cancer-related fatigue syndrome in patients with advanced cancer: a feasibility study. Palliat Med 2006;20:413-418.

15. Fukuda K, Straus SE, Hickie I, et al. The chronic fatigue syndrome: a comprehensive approach to its definition and study. International Chronic Fatigue Syndrome Study Group. Ann Intern Med 1994;121:953-959.

16. Alexander $\mathbf{S}$, Minton 0 , Andrews P, et al. A comparison of the characteristics of disease-free breast cancer survivors with or without cancer-related fatigue syndrome. Eur J Cancer 2009;45:384-392.

17. Ancoli-Israel S, Liu L, Marler MR, et al. Fatigue, sleep, and circadian rhythms prior to chemotherapy for breast cancer. Support Care Cancer 2006;14:201-209.

18. Liu L, Fiorentino L, Natarajan L, et al. Pre-treatment symptom cluster in breast cancer patients is associated with worse sleep, fatigue and depression during chemotherapy. Psychooncology 2009;18:187-194.

19. Correa DD, Ahles TA. Neurocognitive changes in cancer survivors. Cancer $J$ 2008;14:396-400.

20. Servaes P. Relations between fatigue neuropsychological functioning and physical activity after treatment for breast carcinoma. Cancer 2002;95:2017-2026.

21. Cella D. The Functional Assessment of Cancer Therapy-Anemia (FACT-An) Scale: a new tool for the assessment of outcomes in cancer anemia and fatigue. Semin Hematol 1997;34:13-19.

22. Minton 0, Richardson A, Sharpe M, et al. A systematic review and meta-analysis of the pharmacological treatment of cancer-related fatigue. J Natl Cancer Inst 2008; 100:1155-1166.

23. Chalder T, Berelowitz G, Pawlikowska T, et al. Development of a fatigue scale. J Psychosom Res 1993;37:147-153.

24. Minton 0, Stone P. A systematic review of the scales used for the measurement of cancer-related fatigue (CRF). Ann Oncol 2009;20:17-25.

25. NCCN. NCCN: Clinical practice guidelines in oncology. Cancer related fatigue, 2010

26. Zigmond AS, Snaith RP. The hospital anxiety and depression scale. Acta Psychiatr Scand 1983;67:361-370.

27. Stone $\mathbf{P}$, Hardy J, Broadley K, et al. Fatigue in advanced cancer: a prospective controlled cross-sectional study. Br J Cancer 1999;79:1479-1486.

28. Aaronson NK, Ahmedzai S, Bergman B, et al. The European Organization for Research and Treatment of Cancer QLO-C30: a quality-of-life instrument for use in international clinical trials in oncology. J Natl Cancer Inst 1993;85:365-376.

29. Sprangers MA, Groenvold M, Arraras Jl, et al. The European Organization for Research and Treatment of Cancer breast cancer-specific quality-of-life questionnaire module: first results from a three-country field study. J Clin Oncol 1996;14:2756-2768.

30. Weinstein AA, Drinkard BM, Diao G, et al. Exploratory analysis of the relationships between aerobic capacity and self-reported fatigue in patients with rheumatoid arthritis, polymyositis, and chronic fatigue syndrome. PM R 2009;1:620-628. 
31. Savard J, Simard S, Blanchet J, et al. Prevalence, clinical characteristics, and risk factors for insomnia in the context of breast cancer. Sleep 2001;24:583-590.

32. Kushida CA, Chang A, Gadkary C, et al. Comparison of actigraphic, polysomnographic, and subjective assessment of sleep parameters in sleepdisordered patients. Sleep Med 2001;2:389-396.

33. Quesnel C, Savard J, Ivers H. Cognitive impairments associated with breast cancer treatments: results from a longitudinal study. Breast Cancer Res Treat 2009;116:113-123.

34. Servaes $\mathbf{P}$, van der Werf S, Prins J, et al. Fatigue in disease-free cancer patients compared with fatigue in patients with chronic fatigue syndrome. Support Care Cancer 2001;9:11-17.

35. Minton 0, Stone PC. Review: the use of proteomics as a research methodology for studying cancer-related fatigue: a review. Palliat Med 2010;24:310-316.

36. Alexander S, Stone P, White S, et al. Evaluation of central serotonin sensitivity in breast cancer survivors with cancer-related fatigue syndrome. J Pain Symptom Manage 2010;40:892-898.

37. Minton 0, Richardson A, Sharpe M, et al. Drug therapy for the management of cancer-related fatigue. Cochrane Database Syst Rev 2010;7:CD006704.

38. Minton 0, Richardson A, Sharpe M, et al. Psychostimulants for the management of cancer-related fatigue: a systematic review and meta-analysis. J Pain Symptom Manage 2011:41:761-767.

39. Savard J, Simard S, Ivers $\mathrm{H}$, et al. Randomized study on the efficacy of cognitivebehavioral therapy for insomnia secondary to breast cancer, part I: sleep and psychological effects. J Clin Oncol 2005;23:6083-6096.
40. Berger A, Wielgus K, Hertzog M, et al. Patterns of circadian activity rhythms and their relationships with fatigue and anxiety/depression in women treated with breast cancer adjuvant chemotherapy. Support Care Cancer 2010; 18:105-114.

41. Winningham ML, Nail LM, Burke MB, et al. Fatigue and the cancer experience: the state of the knowledge. Oncol Nurs Forum 1994;21:23-36.

42. Cramp $\mathbf{F}$, Daniel J. Exercise for the management of cancer-related fatigue in adults. Cochrane Database Syst Rev 2008;2:CD006145.

43. Storey DJ, Waters RA, Hibberd CJ, et al. Clinically relevant fatigue in cancer outpatients: the Edinburgh Cancer Centre symptom study. Ann Oncol 2007; 18:1861-1869.

44. Jones M, Schenkel B, Just J, et al. Epoetin alfa improves quality of life in patients with cancer: results of metaanalysis. Cancer 2004;101: 1720-1732.

45. Prue G, Rankin J, Allen J, et al. Cancer-related fatigue: A critical appraisal. Eur J Cancer 2006;42:846-863.

46. Armes J, Chalder T, Addington-Hall J, et al. A randomized controlled trial to evaluate the effectiveness of a brief, behaviorally oriented intervention for cancer-related fatigue. Cancer 2007;110:1385-1395.

47. DOH: http://www.dh.gov.uk/en/Publicationsandstatistics/Publications/ PublicationsPolicyAndGuidance/DH_088879 cancer survivorhsip strategy 2008 (accessed 1 May 2012). 